This item was submitted to Loughborough's Research Repository by the author.

Items in Figshare are protected by copyright, with all rights reserved, unless otherwise indicated.

\title{
Broadband MIMO beamforming for frequency selective channels using the sequential best rotation algorithm
}

PLEASE CITE THE PUBLISHED VERSION

PUBLISHER

(c) IEEE

VERSION

VoR (Version of Record)

\section{LICENCE}

CC BY-NC-ND 4.0

\section{REPOSITORY RECORD}

Davies, Martin, Sangarapillai Lambotharan, Jonathon Chambers, and John McWhirter. 2019. "Broadband MIMO Beamforming for Frequency Selective Channels Using the Sequential Best Rotation Algorithm". figshare. https://hdl.handle.net/2134/5541. 
This item was submitted to Loughborough's Institutional Repository (https://dspace.lboro.ac.uk/) by the author and is made available under the following Creative Commons Licence conditions.

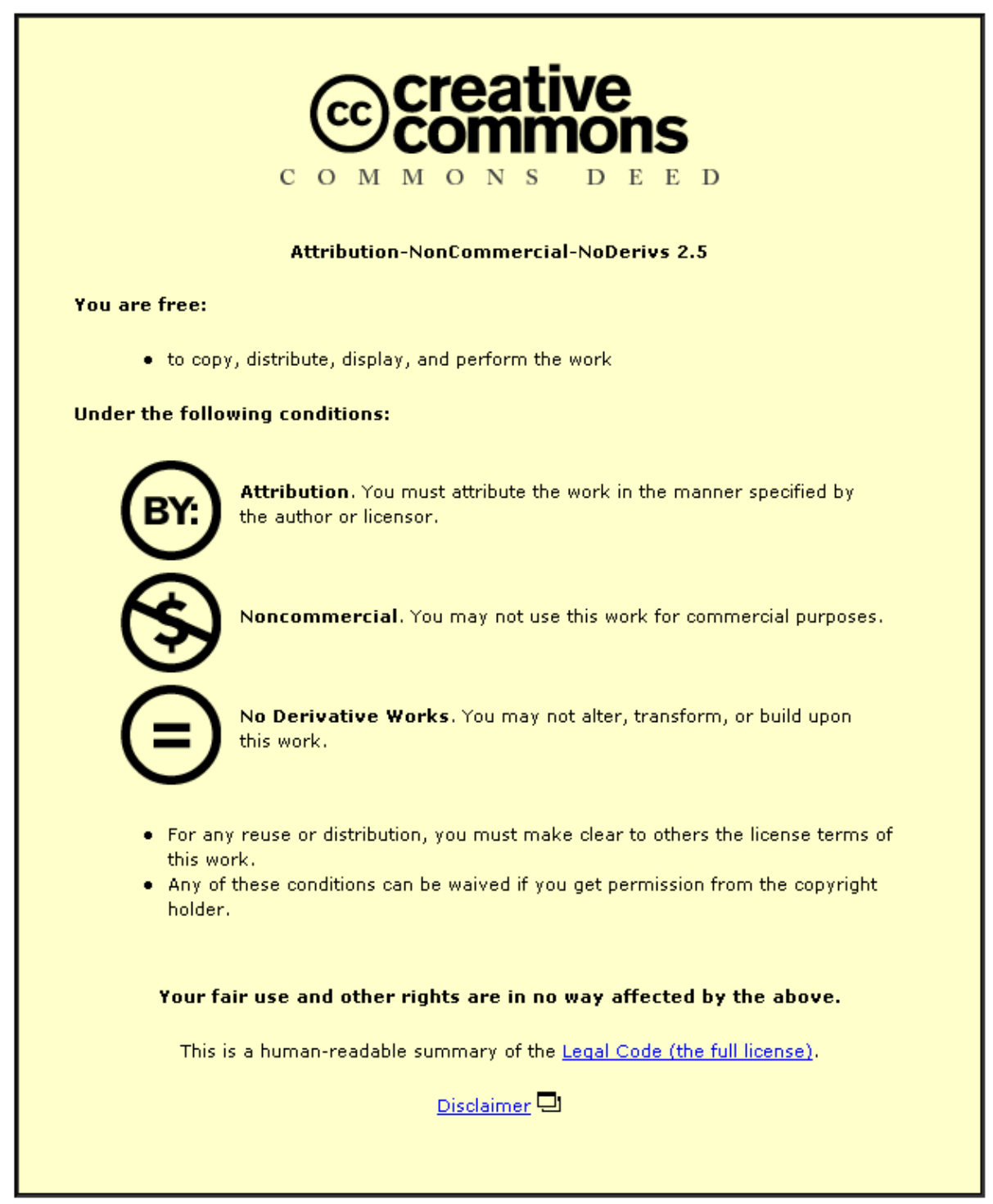

For the full text of this licence, please go to: http://creativecommons.org/licenses/by-nc-nd/2.5/ 


\section{Broadband MIMO Beamforming For Frequency Selective Channels Using The Sequential Best Rotation Algorithm}

\author{
Martin Davies, Sangarapillai Lambotharan \\ and Jonathon Chambers \\ Advanced Signal Processing Group \\ Department of Electronic and Electrical Engineering \\ Loughborough University LE11 3TU, UK \\ Email: \{M.R.Davies, S.Lambotharan, J.A.Chambers\} @lboro.ac.uk
}

\author{
John McWhirter \\ Center for D.S.P \\ School of Engineering \\ Cardiff University CF24 3AA, UK \\ Email: mcwhirterjg@cardiff.ac.uk
}

\begin{abstract}
For a narrowband multi-input multi-output (MIMO) system the singular value decomposition has the ability to provide multiple spatial channels for data transmission. We extend this work to obtain spatial diversity techniques for frequency selective MIMO systems using a polynomial matrix decomposition known as the sequential best rotation using second order statistics (SBR2) method. This algorithm diagonalizes a MIMO frequency selective channel yielding various spatial modes for data transmission. We evaluate the diversity performance of the dominant channel provided by the SBR2 based broadband decomposition and compare it with a transmit antenna selection method (TAS) and a MIMO orthogonal frequency-division multiplexing (OFDM) singular value decomposition (SVD) based approach. Simulation results show SBR2 significantly outperforms the average bit error rate (BER) of TAS, making it very suitable for time division multiple access (TDMA) and code division multiple access (CDMA) systems. SBR2 and MIMO-OFDM systems are shown to have identical BER performance, confirming the efficiency of the proposed low delay spatial-temporal scheme.
\end{abstract}

\section{INTRODUCTION}

For a wireless system consisting of $M_{t}$ transmit antennas and $M_{r}$ receive antennas, the noise free channel can be represented as an $M_{r} \times M_{t}$ matrix, $\boldsymbol{H}$. In a narrowband scenario where the received signals are instantaneously mixed, a scalar matrix is sufficient to describe the mixing. Paulraj, Nabar and Gore [1] have shown that for this scenario, performing the SVD would provide a number of independent spatial subchannels which can be used to enhance the data rates or to obtain increased diversity.

In the case of a broadband MIMO system the noise free multipath channel can be represented as a polynomial matrix, $\underline{\boldsymbol{H}}(z)$

$$
\underline{\boldsymbol{H}}(z)=\sum_{i=0}^{L-1} \boldsymbol{H}_{i} z^{-i}
$$

where $\boldsymbol{H}_{i} \in \mathbb{C}^{M_{r} \times M_{t}}$ is the $i^{t h}$ matrix tap of the MIMO channel of length $L$, and $z^{-i}$ is the unit delay operator. Thus the SVD cannot be applied. A typical approach to the broadband problem is to reduce it to a narrowband form by using a discrete Fourier transform (DFT) to split the broadband channel into narrow uniformly spaced frequency bands and applying the SVD in each band. This is suitable for OFDM based systems. However, in this paper, we propose the polynomial matrix decomposition for obtaining various spatial modes as this is applicable to a range of access schemes such as TDMA and CDMA.

SBR2 is a novel algorithm [2] for calculating the eigenvalue decomposition (EVD) of a polynomial matrix and its application allows a true broadband SVD to be performed on $\underline{\boldsymbol{H}}(z)$. The motivation therefore is to extend the decomposition presented in [1] to a broadband MIMO scenario.

We wish to emphasize that unlike DFT based decompositions (which require storage of data and also incur the associated block delay at the receiver), the proposed scheme operates using spatial-temporal filters in the time domain, hence it is more suitable for decoding continuous streams of data without requiring storage of signals for DFTs and unnecessary block delays. Moreover, its sensitivity to frequency offset errors is likely to be less than for OFDM-schemes.

\section{Channel Model}

In our simulation, without loss of generality we consider a MIMO system with five antennas at the transmitter and receiver. The length of the channel between each transmitter and receiver is five, and the channel coefficients have been assumed to be unchanged within each data block, but allowed to change between data blocks according to a complex Gaussian distribution. An exponential power delay profile has been used for the channel with an exponential decaying factor of $\psi=0.8$, i.e.

$$
h_{i j}(k, n)=\alpha \exp ^{-\psi(k-1)} w(n), k=1, \ldots, 5
$$

where $h_{i j}(k, n)$ is the $k^{\text {th }}$ multipath at time index $n$, between the $i^{\text {th }}$ transmit antenna and $j^{\text {th }}$ receive antenna, $w(n)$ is zero mean complex circular Gaussian noise, and the parameter $\alpha$ is chosen to normalize the channel gain. 


\section{SBR2 ALGORITHM}

The input to the SBR2 algorithm is a para-Hermitian polynomial matrix, which may be expressed as

$$
\underline{\boldsymbol{R}}(z)=\sum_{\tau=-\tau_{\max }}^{\tau_{\max }} z^{-\tau} \boldsymbol{R}(\tau)
$$

where $\boldsymbol{R}(\tau) \in \mathbb{C}^{p \times p}$ with elements

$$
\begin{aligned}
{[\boldsymbol{R}(\tau)]_{k l} } & =r_{k l}(\tau)=r_{l k}^{*}(-\tau) \\
& =[\boldsymbol{R}(-\tau)]_{l k}^{*}, k, l \in\{1,2, \ldots, p\}
\end{aligned}
$$

The SBR2 algorithm will calculate the EVD of $\underline{\boldsymbol{R}}(z)$ as in (5) where $\underline{\boldsymbol{D}}(z)$ is a diagonalized polynomial matrix and $\underline{\boldsymbol{P}}(z)$ is a paraunitary diagonalizing matrix and $\underline{\widetilde{\boldsymbol{P}}}(z)$ is its paraconjugate.

$$
\underline{\boldsymbol{D}}(z)=\underline{\boldsymbol{P}}(z) \underline{\boldsymbol{R}}(z) \underline{\widetilde{\boldsymbol{P}}}(z)
$$

The SVD of the polynomial channel, $\underline{\boldsymbol{H}}(z)$ is shown in (6) where $\underline{\boldsymbol{U}}(z)$ and $\tilde{\boldsymbol{V}}(z)$ are paraunitary matrices, so that $\underline{\widetilde{\boldsymbol{U}}}(z) \underline{\boldsymbol{U}}(z)=\boldsymbol{I}$ and $\underline{\tilde{\boldsymbol{V}}}(z) \underline{\boldsymbol{V}}(z)=\boldsymbol{I}$ and $\underline{\boldsymbol{\Lambda}}(z)$ is a diagonal matrix. By convolving the channel matrix with its paraconjugate we obtain the set of equations in (7).

$$
\underline{\boldsymbol{H}}(z)=\underline{\boldsymbol{U}}(z) \underline{\boldsymbol{\Lambda}}(z) \underline{\tilde{\boldsymbol{V}}}(z)
$$

$$
\begin{aligned}
& \underline{\underline{\boldsymbol{H}}}(z) \underline{\widetilde{\boldsymbol{H}}}(z)=\underline{\boldsymbol{U}}(z) \underline{\boldsymbol{\Lambda}}(z) \underline{\widetilde{\boldsymbol{\Lambda}}}(z) \underline{\widetilde{\boldsymbol{U}}}(z) \\
& \underline{\widetilde{\boldsymbol{H}}}(z) \underline{\boldsymbol{H}}(z)=\underline{\boldsymbol{V}}(z) \underline{\widetilde{\boldsymbol{\Lambda}}}(z) \underline{\boldsymbol{\Lambda}}(z) \underline{\widetilde{\boldsymbol{V}}}(z)
\end{aligned}
$$

Comparing (7) and (5) shows that when $\underline{\boldsymbol{R}}(z)=$ $\underline{\boldsymbol{H}}(z) \underline{\underline{\boldsymbol{H}}}(z), \underline{\widetilde{\boldsymbol{P}}}(z) \equiv \underline{\boldsymbol{U}}(z)$ and when $\underline{\boldsymbol{R}}(z)=\underline{\widetilde{\boldsymbol{H}}}(z) \underline{\boldsymbol{H}}(z)$, $\underline{\widetilde{P}}(z) \equiv \underline{V}(z)$. Hence the SVD of the polynomial channel matrix can be calculated using the SBR2 algorithm.

\section{A. Orthogonal Channels}

By analogy with a conventional narrowband SVD beamformer, the process of passing a signal through a transmit filter bank $\underline{\boldsymbol{v}}_{i}(z)$, where $i$ denotes the column index, the channel $\underline{\boldsymbol{H}}(z)$ and a receive filter bank $\underline{\widetilde{\boldsymbol{x}}}_{i}(z)$, where $i$ denotes the row index, is identical to passing the signal through the appropriate element of $\underline{\boldsymbol{\Lambda}}(z)$, calculated by applying (6). For an $M_{r}=M_{t}$ MIMO system, $\underline{\boldsymbol{\Lambda}}(z)$ will be a diagonal matrix containing $M_{r}$ orthogonal channels. The SBR2 algorithm will order the channels in terms of power, hence $\underline{\lambda}_{11}(z)$ will be the strongest channel and $\underline{\boldsymbol{\lambda}}_{M_{r} M_{r}}(z)$ the weakest. Typically, the dominant channel is spectrally majorized resulting in continuous high gain across the signal bandwidth as shown in Figure 1.

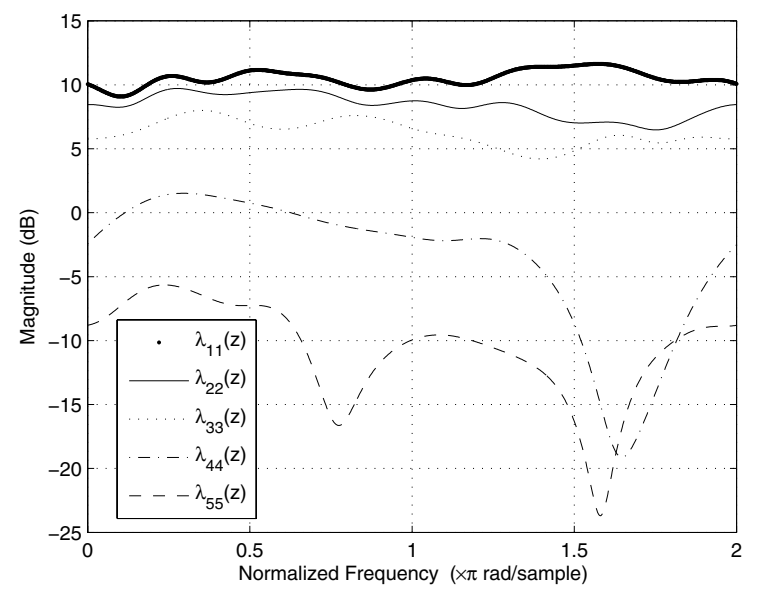

Fig. 1. Typical frequency response of the spatial subchannels designed by SBR2

\section{SBR2 OPERATION}

A real symmetric matrix $\boldsymbol{A}$ can be diagonalized using Jacobi rotation matrices [3]. Initially, the largest in magnitude offdiagonal element of $\boldsymbol{A}$ is located, assumed to be the $p, q^{\text {th }}$ element, i.e. $a_{p q}=[\boldsymbol{A}]_{p q}$. This search can either be in the upper triangular or lower triangular part of $\boldsymbol{A}$ due to its Hermitian property. The rotation angle $\theta$ which is used to annihilate the off-diagonal element $a_{p q}$ of matrix $\boldsymbol{A}$ is chosen such that:

$$
\cot 2 \theta=\frac{a_{q q}-a_{p p}}{2 a_{p q}}
$$

The Jacobi rotation matrix, $\boldsymbol{Q}$ is then given by

$$
\boldsymbol{Q}_{p q} \equiv\left[\begin{array}{ccccccc}
1 & \ldots & 0 & \ldots & 0 & \ldots & 0 \\
\vdots & \ddots & \vdots & & \vdots & & \vdots \\
0 & \ldots & c_{p p} & \ldots & s_{p q} & \ldots & 0 \\
\vdots & & \vdots & \ddots & \vdots & & \vdots \\
0 & \ldots & -s_{q p} & \ldots & c_{q q} & \ldots & 0 \\
\vdots & & \vdots & & \vdots & \ddots & \vdots \\
0 & \ldots & 0 & \ldots & 0 & \ldots & 1
\end{array}\right]
$$

where $c=\cos (\theta)$ and $s=\sin (\theta)$. This matrix is orthogonal by construction. To diagonalize matrix $\boldsymbol{A}$ using Jacobi rotation matrices a sequence of such orthogonal (similarity) transformations are applied of the form:

$$
\boldsymbol{A}^{\prime}=\boldsymbol{Q}_{p q}^{T} \boldsymbol{A} \boldsymbol{Q}_{p q}
$$

Each application of rotation matrices $\boldsymbol{Q}_{p q}$ and $\boldsymbol{Q}_{p q}^{T}$ will zero two elements symmetric in the leading diagonal of $\boldsymbol{A}$ and the sequence of such matrices is chosen so as to eliminate the dominant off-diagonal elements at each step. Successive transformations will partially undo previously zeros, but off 
diagonal terms will eventually tend to zero, resulting in a diagonal matrix $\boldsymbol{D}$, whose elements correspond to the eigenvalues of $\boldsymbol{A}$ :

$$
\boldsymbol{D}=\boldsymbol{Q}_{L}^{T} \ldots \boldsymbol{Q}_{1}^{T} \boldsymbol{A} \boldsymbol{Q}_{1} \ldots \boldsymbol{Q}_{L}
$$

where $L$ is an unspecified number of iterations. SBR2 expands this rotation matrix approach to para-Hermitian polynomial matrices. Initially, the maximum in magnitude offdiagonal coefficient of $\underline{\boldsymbol{R}}(z)$ is located. Once again this search can either be in the upper triangular or lower triangular part of $\underline{\boldsymbol{R}}(z)$ due to its para-Hermitian property. A delay matrix $\underline{\boldsymbol{B}}(z)$ is first applied to shift the dominant coefficient to the plane of order zero. A rotation matrix is then designed to drive the dominant coefficient to zero resulting in $\underline{\boldsymbol{R}}^{\prime}(z)$.

If we denote $\underline{G}(z)$ the product of the rotation matrix $\boldsymbol{Q}$ and the delay matrix $\underline{\boldsymbol{B}}(z)$ then $\underline{\boldsymbol{R}}^{\prime}(z)$ is given explicitly by:

$$
\underline{\boldsymbol{R}}^{\prime}(z)=\underline{\boldsymbol{G}}(z) \underline{\boldsymbol{R}}(z) \underline{\widetilde{\boldsymbol{G}}}(z)
$$

This process can be repeated iteratively until the magnitude of the maximum off-diagonal coefficient is $\approx 0$ or sufficiently small. This is usually determined by the ratio of the maximum off-diagonal element to the diagonal coefficients of the diagonalized matrix [2], in which a proof of convergence is given.

\section{A. Paraunitary Matrices}

Defining the paraunitary matrix $\underline{\widetilde{\boldsymbol{P}}}(z)$ as:

$$
\underline{\widetilde{\boldsymbol{P}}}_{L}(z)=\underline{\widetilde{\boldsymbol{G}}}_{1}(z) \underline{\widetilde{\boldsymbol{G}}}_{2}(z) \ldots \underline{\widetilde{\boldsymbol{G}}}_{L}(z)
$$

where $L$ represents an unspecified number of iterations and therefore after $L$ iterations (12) results in:

$$
\underline{\boldsymbol{D}}_{L}(z)=\underline{\boldsymbol{P}}_{L}(z) \underline{\boldsymbol{R}}(z) \underline{\widetilde{\boldsymbol{P}}}_{L}(z)
$$

allowing a polynomial matrix EVD to be performed. Figure 2 shows a typical para-Hermitian input, $\underline{\boldsymbol{R}}(z)$ to SBR2 formed from an exponential channel. Figure 3 shows the resulting diagonalization, $\underline{\boldsymbol{D}}(z)$.

\section{B. Limiting the order of polynomial matrices within the SBR2 algorithm}

With each delay step, at each iteration of the algorithm, the order of the polynomial matrix being diagonalized increases, often after a series of iterations becoming unnecessarily large. At each iteration new coefficient matrices are created at both ends of the matrix to accommodate the shifted coefficients, which now exceed the order of the initial polynomial matrix[4]. Over a series of iterations the outer coefficient matrices typically contain only a small proportion of the Frobenius norm, $\|\cdot\|_{F}^{2}$ of the initial $\underline{\boldsymbol{R}}(z)$ or often entirely consist of zeros. In the case of a polynomial matrix, $\|\cdot\|_{F}^{2}$ is simply the sum of the squared Frobenius norms for all coefficient matrices.

The effect of this matrix growth is twofold, firstly computational complexity increases considerably and the algorithm

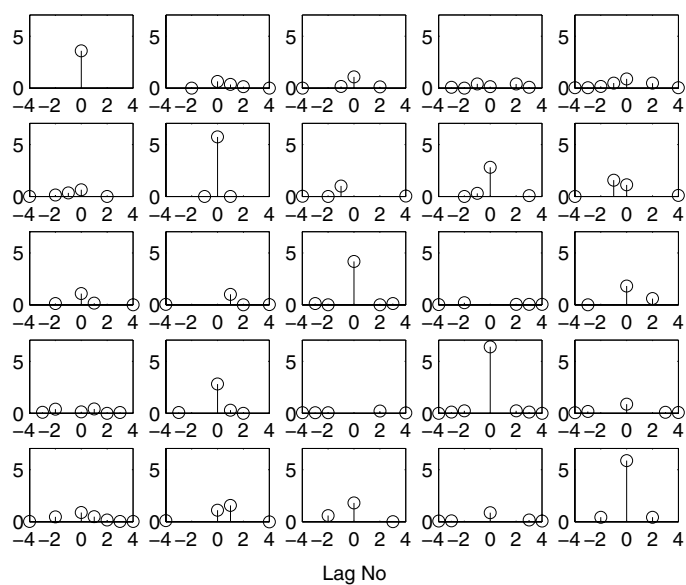

Fig. 2. Coefficients of para-Hermitian matrix $\underline{\boldsymbol{R}}(z)$

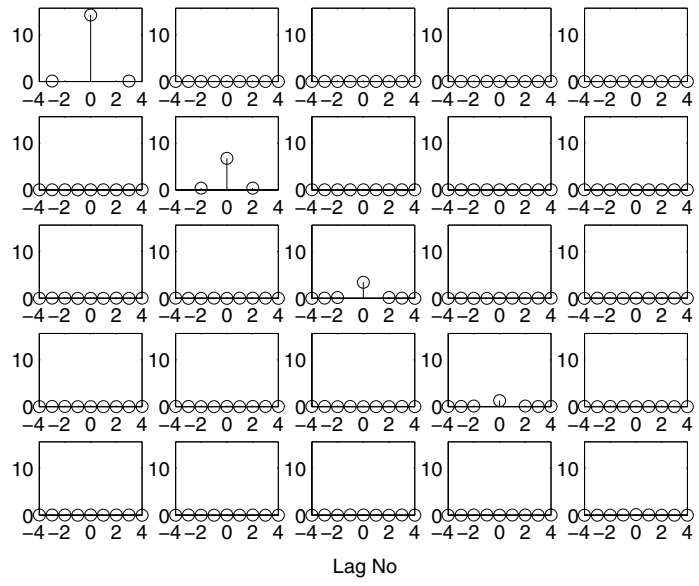

Fig. 3. Coefficients of the diagonalized para-Hermitian matrix $\underline{\boldsymbol{D}}(z)$

becomes too slow to implement in a wireless communications system. Secondly, the memory storage requirements become unnecessarily large. To prevent this we allow a small proportion of the initial Frobenius norm to be lost and trim $\underline{D}(z)$ at every iteration. Let $M$ be the order of $\underline{D}_{i}(z)$ at a given iteration $i$. We find the smallest possible positive value for the lag parameter $t_{l i m}$ such that the following condition holds:

$$
\frac{2 \sum_{t=t_{\text {lim }}}^{M / 2} \sum_{j=1}^{p} \sum_{k=1}^{p}\left|d_{j k}(t)\right|^{2}}{\sum_{-\tau_{\max }}^{\tau_{\max }} \sum_{j=1}^{p} \sum_{k=1}^{p}\left|r_{j k}(\tau)\right|^{2}} \leq 1 \times 10^{-7}
$$

where $d_{j k}$ denotes the $(j, k)^{t h}$ element of the coefficent matrix $\boldsymbol{D}(t)$ and $r_{j k}$ denotes the $(j, k)^{t h}$ element of the input para-Hermitian matrix $\boldsymbol{R}(\tau)$. When a value for $t_{l i m}$ is found, the truncated matrix, $\underline{\boldsymbol{D}}_{i}^{\text {trimed }}(z)$, becomes

$$
\underline{\boldsymbol{D}}_{i}^{\text {trimed }}(z)=\sum_{t=-t_{\text {lim }+1}}^{t_{\text {lim }}-1} \boldsymbol{D}_{i}(t) z^{-t}
$$


Once the algorithm is complete we calculate the paraunitary diagonalizing matrix, $\underline{\boldsymbol{P}}(z)$ from the individual delay and rotation matrices (13). We apply an identical method of truncation as described above to the paraunitary matrix at each iteration.

\section{System IMPLEMENTATION}

The polynomial decomposition provides five spatial subchannels $\lambda_{i i}(z), i=1,2, \ldots, 5$ as shown in Figure 4. We wish to emphasize that unlike in narrowband systems, these spatial subchannels are frequency selective, and further processing is required at the receiver to equalize each subchannel. A typical subchannel impulse response is shown in Figure 5. In our simulation we use a Viterbi equalizer at the receiver to equalize the resulting spatial subchannels. We notice that for the dominant subchannel, most of the energy is concentrated in five consecutive taps. Therefore we extract these significant five taps and pass them to the Viterbi equalizer.

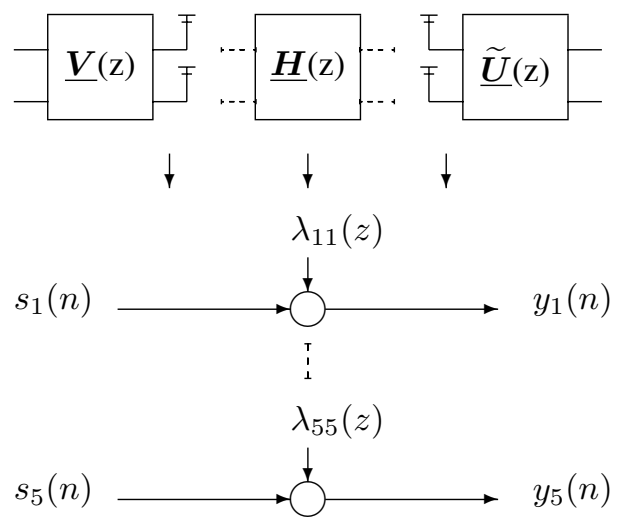

Fig. 4. SBR2 system diagram showing decomposition into five spatial subchannels

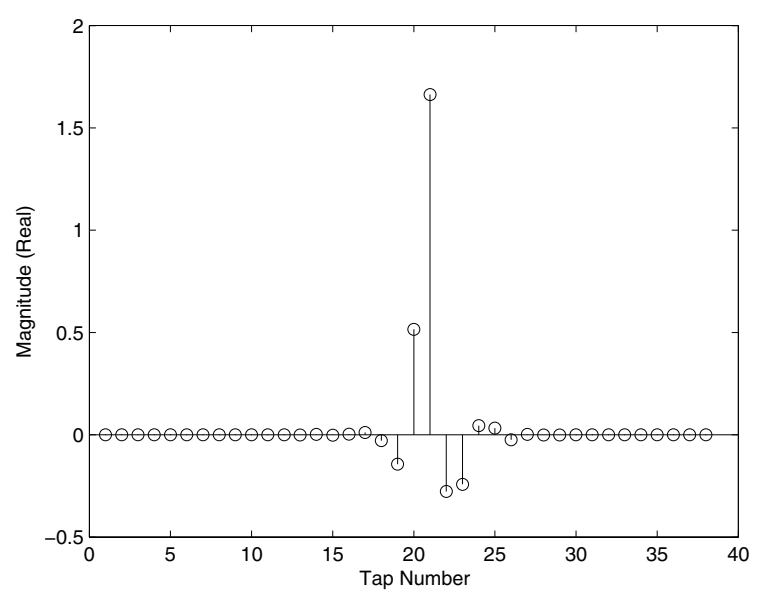

Fig. 5. Typical $\underline{\boldsymbol{\lambda}}_{11}(z)$ stem plot showing a polynomial channel produced by SBR2

\section{A. OFDM scheme}

We have used a MIMO-OFDM scheme as a benchmark. MIMO-OFDM is a DFT based technique that decomposes the otherwise frequency selective channel of bandwidth $B$ into $N$ orthogonal frequency flat MIMO channels, each with bandwidth $B / N$ [1]. The transmitter first performs an inverse fast Fourier transform (IFFT) operation on the signal to be transmitted from each individual transmit antenna. A cyclic prefix (CP) is then added of length $L-1$ prior to transmission. At the receiver the cyclic prefix is stripped off and a FFT applied to the signal received at each antenna.

The cyclic prefix renders the channel impulse response circulant. If we denote $\underline{\boldsymbol{H}}_{c}(z)$ as the circulant channel impulse response then the eigendecomposition of $\underline{\boldsymbol{H}}_{c_{i j}}(z)$, the circulant SISO channel between the $i^{t h}$ transmit antenna and $j^{\text {th }}$ receive antenna may be expressed as

$$
\underline{\boldsymbol{H}}_{c_{i j}}(z)=\boldsymbol{D}^{H} \boldsymbol{\Omega} \boldsymbol{D}
$$

where $D$ represents the unitary discrete Fourier transform matrix and $\boldsymbol{\Omega}=\operatorname{diag}\{\omega[0], \omega[1], \ldots, \omega[N-1]\}$, with

$$
\omega[k]=\sum_{l=0}^{L-1} \boldsymbol{H}_{i j}(l) \exp ^{-\frac{j 2 \pi k l}{N}}
$$

where $k=0,1, \ldots, N-1$ is the sampled frequency response of the SISO channel where $k$ represents the tone index.

Extending this to MIMO, the matrix $\boldsymbol{E}[k]$ is the frequency response of the narrowband MIMO matrix channel corresponding to the $k^{t h}$ tone and is related to $\underline{\boldsymbol{H}}(z)$ by (19).

$$
\boldsymbol{E}[k]=\sum_{l=0}^{L-1} \boldsymbol{H}(l) \exp ^{-\frac{j 2 \pi k l}{N}}
$$

Hence the MIMO frequency selective channel has been decomposed into $N$ MIMO orthogonal frequency flat channels. We then apply the standard SVD within each narrowband tone to provide five spatial subchannels.

\section{Simulations}

The bit error rate has been computed for 1000 Monte Carlo simulations. We have used a TAS scheme as a TDMA benchmark. TAS operates by transmitting exclusively through the best antenna that results in the highest possible SNR at the receiver, creating a single-input multi-output (SIMO) subset of the MIMO system. The received signal at each antenna is then fed into a multiple-input Viterbi equalizer. Identical channels have been used for SBR2, TAS and OFDM methods. The modulation scheme used is BPSK. The number of bits sent, $N=1024$. The total transmission power budgets for the SBR2, TAS and OFDM schemes are identical.

Figure 6 clearly shows the benefit of the SBR2 algorithm. BER performance is far superior to the TAS scheme, for example a $4 \mathrm{~dB}$ reduction in SNR to acheive identical average BER of $10^{-3}$, making SBR2 highly suitable for TDMA and CDMA applications. 


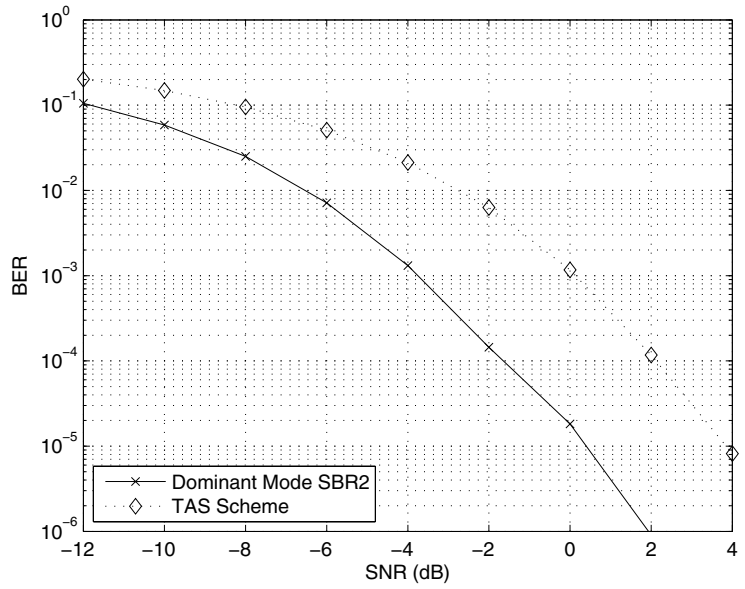

Fig. 6. Average BER results for dominant mode SBR2 and TAS schemes

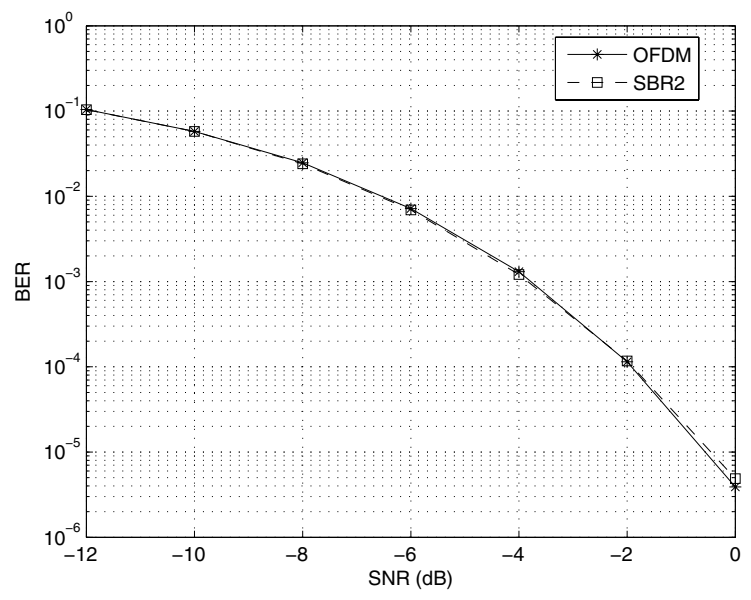

Fig. 7. Average BER results for dominant mode SBR2 and OFDM schemes

Figure 7 shows that both the SBR2 and MIMO-OFDM methods perform identically. OFDM transmission incurs on average a loss in spectral efficiency of $(L-1) /(N+L-1)$ on account of the cyclic prefix. If $N \gg L$, this loss is negligible [1] so this has not been considered. Figure 8 shows the frequency response of the dominant SBR2 and MIMOOFDM mode for one of the channels used. As can be seen both systems have an identical frequency response. The only slight difference in performance is due to the trimming of the polynomial matrices within the SBR2 algorithm. This does not occur within the MIMO-OFDM system as the DFT and SVD operations are inherently lossless.

We have therefore shown that our "continuous" time domain SBR2 approach provides exactly the same performance as the "discrete" frequency domain approach of MIMO-OFDM.

\section{CONCLUSION}

In this paper we have discussed the operation and implementation of SBR2 into a broadband MIMO scenario. By building on the work of Paulraj, Nabar and Gore it is demonstrated

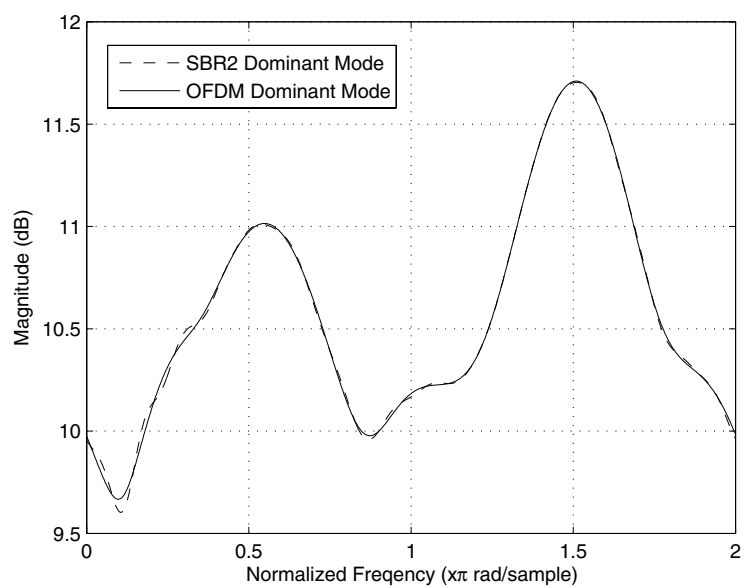

Fig. 8. Frequency response of the dominant mode for SBR2 and MIMOOFDM

that the natural extension of the SVD to polynomial matrices, SBR2 is capable of solving the difficult MIMO broadband problem and provides identical performance to an OFDM based solution. However, as SBR2 operates entirely in the time domain it is potentially suitable for TDMA and CDMA based applications.

We have not discussed the effect of incorrect channel estimation. In an OFDM based system this will destroy the circulant property (17). Hence the $N$ flat fading tones will no longer be orthogonal and inter-symbol interference (ISI) will occur between the tones. Due to the Viterbi equalization strategy used in the SBR2 system, SBR2 will be less susceptible to subchannel interference. Additionally in a scenario where accurate channel estimations are unobtainable, only the dominant mode of SBR2 can be used with a whitening transformation added to widen the beam width. By using only the dominant mode this will mitigate the effects of interference from other SBR2 modes. Using only the dominant mode in a OFDM system will not remove ISI between tones leading to unavoidable poor system performance.

\section{ACKNOWLEDGMENT}

The authors would like to thank Dr Stephan Weiss and Professor Ian Proudler for their invaluable comments and suggestions. The work of Martin Davies is jointly funded by EPSRC and QinetiQ.

\section{REFERENCES}

[1] A. Paulraj, R. Nabar, and D. Gore, Introduction to Space-Time Wireless Communications, 1st ed. University Press, Cambridge: Cambridge University Press, 2003.

[2] J. McWhirter, P. Baxter, T. Cooper, S. Redif, and J. Foster, "An EVD algorithm for para-Hermitian polynomial matrices," IEEE Transactions on Signal Processing, vol. 55, no. 5, pp. 2158-2169, May 2007.

[3] G. Golub and C. Loan, Matrix Computations, 3rd ed. Baltimore, Maryland: The John Hopkins University Press, 1996.

[4] J. Foster, J. McWhirter, and J.Chambers, "Limiting the order of polynomial matrices within the SBR2 algorithim," in Conference Digest: IMA Mathematics in Signal Processing, Cirencester, England, Dec 2006, pp. 93-97. 